\title{
Current regulators for I/SUP 2/L circuits to be operated from low-voltage power supplies
}

Bruun, Erik; Hansen, Ole

Published in:

I E E E Journal of Solid State Circuits

Link to article, DOI:

10.1109/JSSC.1980.1051472

Publication date:

1980

Document Version

Publisher's PDF, also known as Version of record

Link back to DTU Orbit

Citation (APA):

Bruun, E., \& Hansen, O. (1980). Current regulators for I/SUP 2/L circuits to be operated from low-voltage power supplies. I E E E Journal of Solid State Circuits, 15(5), 796-799. https://doi.org/10.1109/JSSC.1980.1051472

\section{General rights}

Copyright and moral rights for the publications made accessible in the public portal are retained by the authors and/or other copyright owners and it is a condition of accessing publications that users recognise and abide by the legal requirements associated with these rights.

- Users may download and print one copy of any publication from the public portal for the purpose of private study or research.

- You may not further distribute the material or use it for any profit-making activity or commercial gain

- You may freely distribute the URL identifying the publication in the public portal

If you believe that this document breaches copyright please contact us providing details, and we will remove access to the work immediately and investigate your claim 


\title{
Current Regulators for $\mathrm{I}^{2} \mathrm{~L}$ Circuits to be Operated from Low-Voltage Power Supplies
}

\author{
ERIK BRUUN, MEMBER, IEEE, AND OLE HANSEN, MEMBER, IEEE
}

\begin{abstract}
A new bandgap current reference is described which can be used to control the injector current of $\mathrm{I}^{2} \mathbb{L}$ circuits for supply voltages down to about $1 \mathrm{~V}$. For small currents the total injector current is obtained as a mirror of the reference current. For large injector currents the current control is performed by a series regulator which compares the injector current of one $I^{2} L$ gate to the reference current.

The described reference current can be adjusted to give a variation with temperature of about $60 \mathrm{ppm} /{ }^{\circ} \mathrm{C}$ over the temperature range -10 to $+70^{\circ} \mathrm{C}$. However, in some applications a nonzero, but well controlled temperature coefficient is desired. It is shown how a temperature stable ring oscillator with $\mathbf{I}^{2} \mathrm{~L}$ gates can be constructed by tailoring the temperature dependence of the supply current appropriately.
\end{abstract}

\section{INTRODUCTION}

$\mathrm{O}$ NE OF the potential advantages of $\mathrm{I}^{2} \mathrm{~L}$ circuits is that they can, in principle, be operated from supply voltages less than $1 \mathrm{~V}$. This permits, e.g., the use of a single NiCad cell as the power supply -an attractive feature in such applications as camera controls, watches, and calculators. However, some means for controlling the injector current are required. Normally a series drop resistor $R_{c}$ is connected from the supply voltage $V_{c c}$ to the injector in which case the total injector current is

$$
I_{\mathrm{inj}}=\frac{V_{c c}-V_{B E}}{R_{c}} .
$$

When $V_{c c}$ approaches $V_{B E}$ this solution becomes useless due to the temperature dependence of the base-emitter voltage $V_{B E}$. Also variations in $V_{c c}$ give rise to large relative variations in $I_{\text {inj }}$ when $V_{c c}$ is only slightly larger than $V_{B E}$.

These problems have led to the investigation of principles for implementation of a current control circuit which can be used with input voltages down to about $V_{B E}$.

In the present paper we first discuss the circuit design of a constant reference current source which can operate down to less than $1 \mathrm{~V}$. The current source is implemented using a bandgap reference principle, aiming at a temperature coefficient of zero. As the current is sourced to the load through a lateral p-n-p transistor it is only possible to use it directly as the injector current supply for supply currents below approxi-

Manuscript received March 20, 1980; revised May 30, 1980.

E. Bruun was with the Electronics Institute, Laboratory for Semiconductor Technology, Technical University of Denmark, Lyngby, Denmark. He is now with Christian Rovsing A/S, Lautrupvang 2, Ballerup, Denmark.

O. Hansen is with the Electronics Institute, Laboratory for Semiconductor Technology, Technical University of Denmark, Lyngby, Denmark. mately $1 \mathrm{~mA}$. However, for larger values of injector current the described current source can be used as a reference current in a feedback arrangement which employs an external p-n-p transistor as the series pass element.

While the primary aim in the design of the reference current source has been the establishment of a temperature stable current source operable down to about $1 \mathrm{~V}$ the demand in some $\mathrm{I}^{2} \mathrm{~L}$ circuits may be a current source that exhibits a nonzero, but well controlled variation with temperature in order to get a constant gate delay. We, therefore, show how the temperature coefficient of the reference current can be adjusted such that a very small variation with temperature of the oscillator frequency of a three-stage ring oscillator can be achieved.

\section{Constant Current Source}

A conventional bandgap reference voltage is established by adding a base-emitter voltage of a transistor to a base-emitter voltage difference of two transistors with different emitter current densities. The base-emitter voltage has a negative temperature coefficient while the base-emitter voltage difference has a positive temperature coefficient. It can be shown that if the base-emitter voltage difference is adjusted such that the sum of the two voltages equals the silicon bandgap voltage a zero temperature coefficient of the sum results [1].

When implementing a constant current source the same principle can be applied by using the sum of a $V_{B E}$ dependent current and a $\Delta V_{B E}$ dependent current as the reference current [2].

Fig. 1 shows the diagram of the current source. The reference current is obtained by adding the currents $I_{2}$ and $I_{R}$. The current $I_{R}$ is equal to $V_{B E 1} / R_{1}$ and, thus, has a negative temperature coefficient.

The current $I_{2}$ is equal to $\left(V_{B E 1}-V_{B E 2}\right) / R_{2}$ and has a positive temperature coefficient. The transistors $Q_{3}$ and $Q_{4}$ provide the feedback amplifier which regulates the currents in $Q_{1}, Q_{2}$, and $R_{1}$ such that $I_{\text {ref }}=I_{1}=I_{2}+I_{R}$. The resistor $R_{s}$ is included to ensure a correct start-up of the circuit when the supply voltage is applied, and the capacitor $C$ is added for stability reasons. The reference current is obtained from an extra collector of the lateral p-n-p transistor $Q_{4}$. The reference current is, thus, a source current which can be used directly to supply $\mathrm{I}^{2} \mathrm{~L}$ gates provided that the total current consumption does not exceed the maximum current capability of the transistor $Q_{4}$.

Alternatively a reference sink current can be obtained by adding the transistor $\underline{Q}_{5}$. 


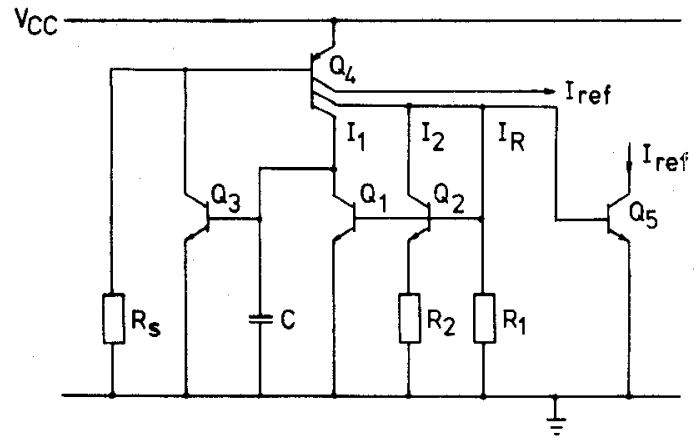

Fig. 1. Schematic of reference current generator.

The temperature dependence of the reference current can be analyzed by examining the currents $I_{2}$ and $I_{R}$. The baseemitter voltage dependence on temperature is given by [1]

$$
\begin{aligned}
V_{B E}= & V_{g 0}\left(1-\frac{T}{T_{0}}\right)+\frac{T}{T_{0}} V_{B E 0} \\
& +V_{t 0} \frac{T}{T_{0}}\left((m-4) \ln \frac{T}{T_{0}}+\ln \frac{I_{c}}{I_{0}}\right) .
\end{aligned}
$$

In (2) $V_{g 0}$ is the silicon bandgap voltage at absolute zero $\left(V_{g 0}=1185 \mathrm{mV}\right), T_{0}$ is the room temperature $\left(T_{0}=300 \mathrm{~K}\right)$, $V_{B E 0}$ is the base-emitter voltage at room temperature and current $I_{0}, V_{t 0}=k T_{0} / q=25.8 \mathrm{mV}, m$ is a constant related. to the base minority carrier mobility ( $m \simeq 1$ for double diffused $\mathrm{n}$-p-n transistors), and $I_{0}$ is the collector current at room temperature $T_{0}$. Hence, we achieve

$$
\begin{aligned}
I_{R}= & \frac{V_{B E 1}}{R_{1}}=\frac{1}{R_{1}}\left(V_{g 0}\left(1-\frac{T}{T_{0}}\right)+\frac{T}{T_{0}} V_{B E 0}\right. \\
& \left.+V_{t 0} \frac{T}{T_{0}}\left((m-4) \ln \frac{T}{T_{0}}+\ln \frac{I_{1}}{I_{0}}\right)\right)
\end{aligned}
$$

and

$$
I_{2}=\frac{V_{B E 1}-V_{B E 2}}{R_{2}}=\frac{V_{t 0}}{R_{2}} \frac{T}{T_{0}}\left(\ln N+\ln \frac{I_{1}}{I_{2}}\right)
$$

In (4) $N$ equals the emitter area ratio of $Q_{2}$ and $Q_{1}$ :

$$
N=\frac{A_{2}}{A_{1}} \text {. }
$$

By differentiation we achieve

$$
\left.\frac{d I_{2}}{d T}\right|_{T=T_{0}}=\frac{I_{20}}{T_{0}} \frac{\ln N+\ln z}{1+\ln N+\ln z}
$$

and

$$
\left.\frac{d I_{R}}{d T}\right|_{T=T_{0}}=-\frac{V_{g 0}-V_{R E 0}-V_{t 0}(m-4)}{R_{1} T_{0}} .
$$

In (6) and (7) we have introduced

$$
z=\left.\frac{I_{1}}{I_{2}}\right|_{T=T_{0}}=\frac{I_{10}}{I_{20}} .
$$

Consequently, we obtain a temperature coefficient of zero if

$$
\frac{1}{z-1} \frac{\ln N+\ln z}{1+\ln N+\ln z}=\frac{V_{g 0}-V_{B E 0}-V_{t 0}(m-4)}{V_{B E 0}} .
$$

This equation determines the relation between $N$ and $z$. The values of $R_{1}$ and $R_{2}$ are determined by

$$
R_{1}\left(I_{10}-I_{20}\right)=V_{B E 0} \Rightarrow R_{1}=\frac{V_{B E 0}}{I_{\text {ref }}} \frac{z}{z-1}
$$

and

$$
R_{2} I_{2}+V_{B E 2}=V_{B E 1} \Rightarrow R_{2}=\frac{z V_{t 0}}{I_{\text {ref }}}(\ln z+\ln N) .
$$

Furthermore, it can be shown that the second derivative of the reference current with respect to temperature can be approximated by

$$
\begin{aligned}
\left.\frac{T_{0}^{2}}{I_{\mathrm{ref}}} \frac{d^{2} I_{\mathrm{ref}}}{d T^{2}}\right|_{T=T_{0}} \simeq & \frac{V_{t 0}(m-4)}{V_{g_{0}}-V_{t}(m-4)} \\
& -\frac{2+\ln N+\ln z}{(1+\ln N+\ln z)^{2}(z(1+\ln z+\ln N)-1)}
\end{aligned}
$$

When the area ratio $N$ approaches infinity this expression reduces to the results found for a conventional bandgap voltage reference [3].

$$
\left.\frac{T_{0}^{2}}{V_{\mathrm{ref}}} \frac{d^{2} V_{\mathrm{ref}}}{d T^{2}}\right|_{T=T_{0}}=\frac{V_{t 0}(m-4)}{V_{g 0}-V_{t_{0}}(m-4)}
$$

For a realistic value of $N$ the bandgap reference described above gives a second-order temperature dependence which is slightly larger than the conventional bandgap reference. As an example we find the second derivative for an area ratio of 18 to be $-9 \cdot 10^{-2}$ compared to the value $-6 \cdot 10^{-2}$ found for the conventional bandgap reference.

In the above calculations we have assumed that the resistors $R_{1}$ and $R_{2}$ are temperature independent. If these resistors have a nonzero temperature coefficient

$$
\alpha=\left.\frac{1}{R} \frac{d R}{d T}\right|_{T=T_{0}}
$$

it can be shown that the design equation (9) should be modified to

$$
\begin{aligned}
& \frac{1}{z-1} \frac{\ln N+\ln z}{1+\ln N+\ln z}=\frac{1}{1-\alpha T_{0}} \\
& \cdot\left(\frac{V_{g 0}-V_{B E 0}-V_{t 0}(m-4)}{V_{B E 0}}+\alpha T_{0}\right) .
\end{aligned}
$$

The circuit in Fig. 1 has been implemented using Exar $\mathrm{I}^{2} \mathrm{~L}$ design kit parts XR-C407 and XR-C408.

Aiming at a reference current of $100 \mu \mathrm{A}$ we find $R_{1}=$ $13.130 \mathrm{k} \Omega$ and $R_{2}=1.620 \mathrm{k} \Omega$.

Fig. 2 shows measured results of $I_{\text {ref }}$ versus the supply voltage $V_{c c}$ at different values of the ambient temperature $T_{a}$. The measurements were taken with the resistors $R_{1}$ and $R_{2}$ 


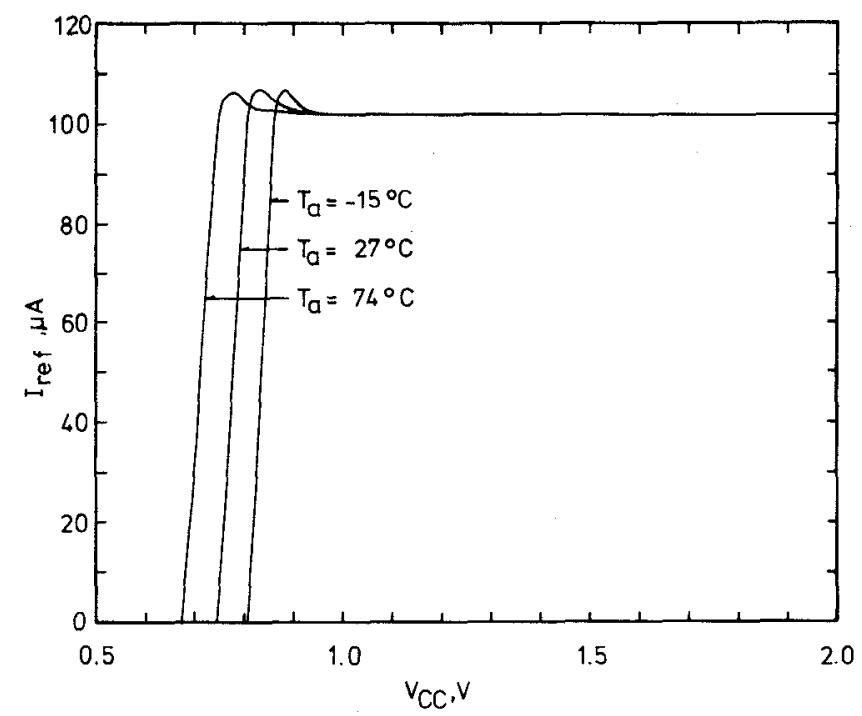

Fig. 2. Reference current $I_{\text {ref }}$ versus supply voltage $V_{c c}$ at different ambient temperatures $T_{a}$.

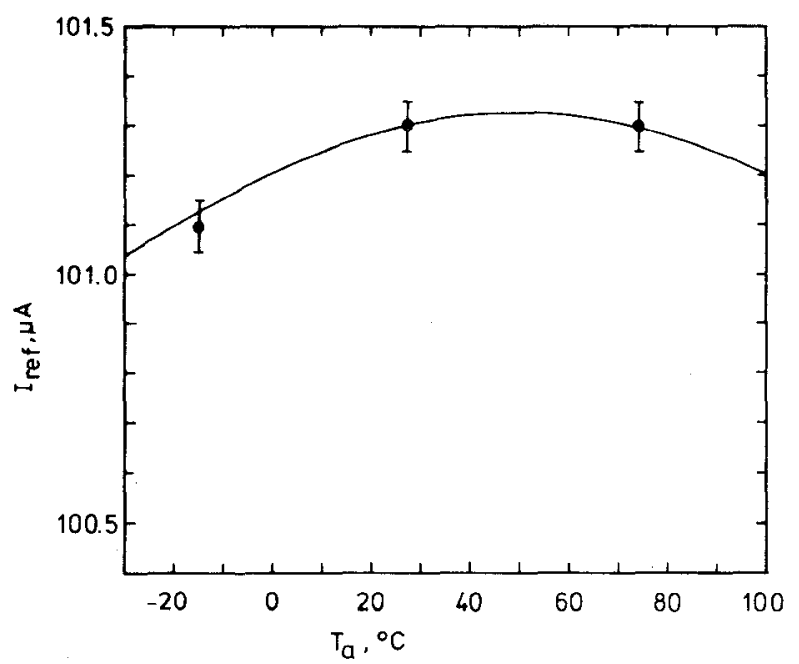

Fig. 3. Calculated and measured temperature dependence of the reference current. For the measured results the measuring uncertainty is indicated in the figure. The measurements were taken with a supply voltage $V_{c c}=1 \mathrm{~V}$.

held at a constant temperature in order to avoid variations in $I_{\text {ref }}$ due to a nonzero temperature coefficient of the resistors. It is evident from Fig. 2 that the circuit is operable below $1 \mathrm{~V}$ over the full temperature range from -10 to $+70^{\circ} \mathrm{C}$. It is also obvious that the reference current exhibits only a very small variation with the supply voltage. The temperature dependence of the reference current is shown in more detail in Fig. 3 where we have shown the calculated performance (a second derivative of $-9 \cdot 10^{-2}$ ) together with the measured results. The measured results are found to be in excellent agreement with the theory.

\section{Feedback Circuit for High Injector Currents}

The current source in Fig. 1 requires the use of a lateral p-n-p transistor as the series pass element. Therefore, the circuit can only be used directly for injector currents below approximately $1 \mathrm{~mA}$. For high current levels an external $\mathrm{p}-\mathrm{n}-\mathrm{p}$

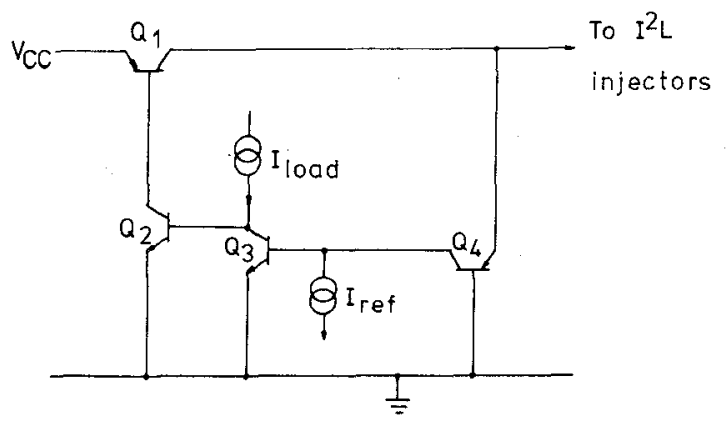

Fig. 4. Series current regulator for high levels of injector current.

series transistor must be used, and the current mirror technique utilized in the circuit in Fig. 1 becomes useless. Instead a feedback arrangement as shown in Fig. 4 may be applied. In this circuit the current reference generated by the circuit in Fig. 1 is compared to the injector current of just one $I^{2} L$ gate. Transistor $Q_{4}$ is, in fact, an $\mathrm{I}^{2} \mathrm{~L}$ gate used as a p-n-p transistor. The collector of $Q_{4}$ is the normal base input of an $\mathrm{I}^{2} \mathrm{~L}$ gate. Hence, the collector current of $Q_{4}$ equals $\alpha_{\text {inj }} \cdot I_{\text {inj }}$ where $\alpha_{\mathrm{inj}}$ is the common base current gain of $Q_{4}$. Assuming a negligible base current of $Q_{3}$ we achieve

$$
\alpha_{\mathrm{inj}} \cdot I_{\mathrm{inj}}=I_{\mathrm{ref}} .
$$

In (15) $I_{\text {inj }}$ is the injector current per gate. The base current of $Q_{3}$ is negligible compared to $I_{\text {ref }}$ if the load current of $Q_{3}$ is of the same order of magnitude as $I_{\mathrm{ref}}$. This can be obtained by taking $I_{10 a d}$ from an extra collector of the p-n-p transistor in the current reference circuit in Fig. 1.

\section{Temperature Stable Ring Oscillator}

A convenient method for implementation of an on-chip oscillator in $\mathrm{I}^{2} \mathrm{~L}$ technique is the use of a chain of inverters as a ring oscillator. As the gate delay of the inverters depends on the injector current the oscillation frequency can be controlled by controlling the injector current. The oscillation frequency is also temperature dependent with the dependency being mainly due to the temperature dependency of the voltage swing.

To a first-order approximation the temperature dependence can be assumed to be linear, and the relative shift in frequency versus temperature can be assumed to be $2 \mathrm{mV} / V_{B E}$ or about 0.3 percent $/{ }^{\circ} \mathrm{C}$.

The oscillation frequency versus temperature is shown in Fig. 5 for a three-stage ring oscillator supplied by a constant current. The oscillator has been implemented using the XR-C401 test circuit.

The temperature dependence can be compensated for by using a $V_{B E}$-dependent injector current or rather $\alpha_{\mathrm{inj}} \cdot I_{\mathrm{inj}}$ should vary in proportion to $V_{B E}$. Such a variation of $\alpha_{\mathrm{inj}}$. $I_{\text {inj }}$ can be obtained by controlling the injector current by the circuit shown in Fig. 4 and using a $V_{B E^{-}}$dependent reference current source.

A $V_{B E}$-dependent reference current source operable from a supply voltage of less than $1 \mathrm{~V}$ can easily be obtained by omitting the transistor $Q_{2}$ and the resistor $R_{2}$ from the reference current generator shown in Fig. 1. The oscillator performance achievable using this approach is also demonstrated in Fig. 5 


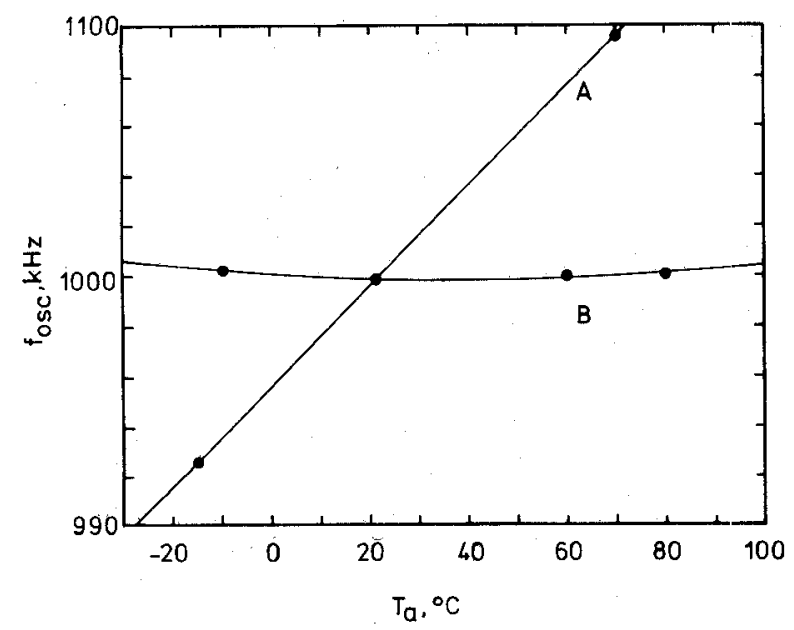

Fig. 5. Oscillation frequency versus temperature for a three-stage ring oscillator supplied from: (a) a constant current source, (b) a series regulator as shown in Fig. 4 and with a $V_{B E^{-}}$dependent reference current.

which shows the oscillation frequency versus temperature for a ring oscillator the injector current of which is controlled by the circuit in Fig. 4 using a $V_{B E}$-dependent reference current.

\section{CONCLUSION}

We have demonstrated a circuit configuration which can be used as a constant current reference. The circuit can be operated from supply voltages below $1 \mathrm{~V}$ and exhibits a temperature stability comparable to the stability achievable from a conventional bandgap voltage reference. The current reference requires two precision resistors for setting of the current level and an external capacitor for stability reasons.

The described current source can directly supply $\mathrm{I}^{2} \mathrm{~L}$ circuits with an injector current up to current levels of about $1 \mathrm{~mA}$. For larger current levels a feedback circuit employing an external $\mathrm{p}-\mathrm{n}-\mathrm{p}$ transistor is proposed. This circuit regulates the current $\alpha_{\text {inj }} \cdot I_{\text {inj }}$ where $I_{\text {inj }}$ is the injector current per gate and $\alpha_{\mathrm{inj}}$ is the common base gain of the injector p-n-p-transistor.

We have finally found that this feedback control of $\alpha_{\mathrm{inj}} \cdot I_{\mathrm{inj}}$ can be used to obtain a temperature independent oscillation frequency from a ring oscillator if $\alpha_{\mathrm{inj}}, I_{\mathrm{inj}}$ is made to vary in proportion to $V_{B E}$. This variation of $\alpha_{\mathrm{inj}} \cdot I_{\mathrm{inj}}$ is obtained from a simplified version of the temperature stable current ref- erence circuit. The oscillator thus implemented shows a variation of frequency of less than $70 \mathrm{ppm} /{ }^{\circ} \mathrm{C}$ over the temperature range from -10 to $+70^{\circ} \mathrm{C}$.

\section{REFERENCES}

[1] R. J. Widlar, "New developments in IC voltage regulators," IEEE J. Solid-State Circuits, vol. SC-6, pp. 2-7, Feb. 1971.

[2] R. J. van de Plassche, "Dynamic element matching for highaccuracy monolithic D/A converters," IEEE J. Solid-State Circuits, vol. SC-11, pp. 795-800, Dec. 1976.

[3] A. P. Brokaw, "A simple three-terminal IC bandgap reference," IEEE J. Solid-State Circuits, vol. SC-9, pp. 388-393, Dec. 1974.

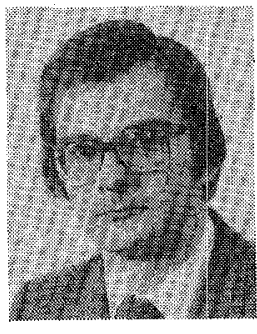

Erik Bruun (S'73-M'74) was born in Copenhagen, Denmark, on December 26, 1949 . He received the M.S. and Ph.D. degrees in electrical engineering from the Technical University of Denmark, Lyngby, Denmark, in 1974 and 1980 , respectively.

From January 1974 to September 1974 he was with the Advanced Systems Department of Christian Rovsing A/S, working on the design of satellite flight-hardware and test-equipment. From September 1974 to April 1980 he was with the Laboratory for Semiconductor Technology, Technical University of Denmark, where he has worked in the areas of MNOS memories, custom-design methodologies, and $\mathrm{I}^{2} \mathrm{~L}$ master slice gate arrays. In April 1980 he rejoined Christian Rovsing A/S where he is presently head of the Microelectronics Branch. He is responsible for the development of custom-designed monolithic integrated circuits and the design and production of hybrid thick film integrated circuits. Since $1979 \mathrm{Mr}$. Bruun has also been coeditor of the Danish professional electronics journal Elektronik.

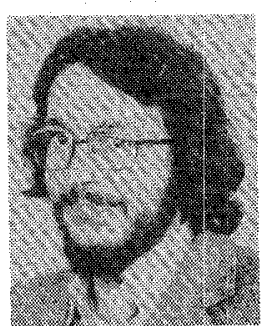

Ole Hansen (S'74-M'76) was born in Sor $\phi$, Denmark, on February 11, 1951. He received the M.S. degree from the Technical University of Denmark, Lyngby, Denmark, in 1977. Presently he is pursuing the Ph.D. degree at the Laboratory for Semiconductor Technology, Technical University of Denmark.

At the Technical University of Denmark he has worked primarily on $I^{2} \mathrm{~L}$ and on epitaxial growth of silicon. He has also performed research in the fields of $I^{2} L$ master-slice gate arrays and n-channel depletion load MOS technology. 